\title{
Effect of thromboplastin and coagulometer interaction on the precision of the International Normalised Ratio
}

\author{
D W Pi, J M Raboud, C Filby, C J Carter
}

\begin{abstract}
Aims-To examine the magnitude of thromboplastin and coagulometer interactions on the precision of International Normalised Ratio (INR) values when the manufacturers' recommended instrument specific International Sensitivity Index (ISI) values are adopted for the INR calculation.

Methods-The variability of INR values obtained from four automated photooptical coagulometers frequently used in North American laboratories was studied. When used with five commercial thromboplastins of moderate to high sensitivity (ISI values $0.92-1.97$ ), 20 prothrombin time results were generated for each of a population of 98 patients on established warfarin treatment.

Results-The mean INR values of the patients ranged from 2.05 to $2 \cdot 81$, depending on which reagent/coagulometer combination was used. Within patient variation increased as the median INR value increased. The mean coefficient of variation of within patient INR values was $10 \%$; the mean coefficient of variation of the prothrombin time results in seconds and prothrombin time ratio were 21 and $18 \%$, respectively.

Conclusions-There was considerable bias in the estimated ISI values of the thromboplastins compared with the manufacturers' instrument specific ISI value. Despite this apparent imperfection, our study clearly showed that the INR is preferable to other prothrombin time reporting formats for assessing the degree of anticoagulation for patients on warfarin treatment.

$(\Im$ Clin Pathol 1995;48:13-17)
\end{abstract}

Keywords: Prothrombin time, anticoagulants, quality control.

International Normalised Ratio (INR) is a standardised method of reporting prothrombin time which compensates for the difference in responsiveness of different thromboplastin reagents used in the prothrombin time assay. The INR is calculated by raising the pro- thrombin time ratio (prothrombin time of patient over mean prothrombin time of normal subjects) to the power of the International Sensitivity Index (ISI) of the thromboplastin reagent. The ISI is the gradient of a logarithmic regression between the specific thromboplastin reagent in use and an international reference thromboplastin preparation (IRP). Although good inter-laboratory precision of INR values could be achieved in laboratories using manual prothrombin time assays, ${ }^{12}$ several studies have revealed that considerable inaccuracy in the INR values may occur when the prothrombin time assays are performed on automated coagulation instruments. ${ }^{34}$ In an effort to minimise the variable effects of thromboplastin and instrument interaction caused by the use of automated coagulation analyser systems in clinical laboratories, major suppliers of commercial thromoboplastins in North America now supply user laboratories with an instrument specific ISI (IS-ISI) value for INR calculation. Unlike the "true" ISI value of a thromboplastin, which requires full calibration according to the World Health Organisation (WHO) protocol using the relevant IRP with the manual technique, the ISISI value is derived from thromboplastin calibration against an IRP using a prototype instrument, similar in design to the one used by the customer's laboratory. ${ }^{56}$ However, the potential benefit of this approach is still questionable ${ }^{78}$ and the degree of INR variability induced by laboratory automation on the clinical care of patients undergoing warfarin treatment remains uncertain. ${ }^{9}$

The purpose of the present study was to examine the magnitude of thromboplastin and coagulometer interactions on the precision of INR values, when the manufacturers' recommended IS-ISI values are adopted for the INR calculation. We must emphasise, however, that because only one instrument of each model of coagulometer was used, our study cannot account for the variable effects of thromboplastin ISIs on individual instruments. In this study five commercial thromboplastins and four models of photo-optical coagulometers frequently used in clinical laboratories in North America were chosen for evaluation. \\ 10 May 1994
}


Table 1 Coagulometers and reagents used in the study

\begin{tabular}{lllll}
\hline Manufacturer & Instrument & Reagent & ISI value & Lot No. \\
\hline Dade, Baxter Diagnostic & MLA Electra 750 & Thromboplastin C Plus & 1.96 & TPC-20A \\
$\quad$ Miami, Florida, USA) & MLA Electra 900C & Thromboplastin IS & 1.33 & TPS-44 \\
& & Innovin & 0.92 & TFS-12 \\
$\begin{array}{l}\text { Organon Teknika } \\
\text { (Scarborough, Ontario, Canada) }\end{array}$ & Coag-a-Mate X2 & Simplastin Excel & 1.97 & 102142 \\
& Coag-a-Mate XM & Simplastin Excel S & $1 \cdot 28$ & 102524 \\
\hline
\end{tabular}

\section{Methods}

The study population comprised 98 outpatients, 55 men and 43 women with a mean age of 62 years (range 44-84 years) on warfarin treatment for more than two months. Prothrombin time assays were performed on the patients' plasma by four photooptical instruments using five commercial thromboplastins with moderate and low ISI values (table 1). These instruments were selected because a recent local survey (unpublished) had shown that they were the most common models used in clinical laboratories. To obtain an estimate of the prothrombin time in the normal population, 22 healthy employees ( 11 men and 11 women) of a clinical laboratory with no history of liver disease or alcoholism were invited to participate in the study. The mean prothrombin time values of these healthy subjects were used to determine the prothrombin time ratios gathered from each of the 20 prothrombin time test systems (instrument/thromboplastin combinations). Citrated venous blood samples (18 $\mathrm{ml}$ ) were collected in vacutainer tubes containing $1.129 \mathrm{M} 3 \cdot 8 \%$ buffered citrated solution (Vacutainer, Becton Dickinson, Franklin Lakes, New Jersey, USA) from both the patients and healthy subjects and were transported, centrifuged and stored according to the National Committee for Clinical Laboratory Standards guidelines. ${ }^{10}$ The plasma samples were aliquoted and frozen at $-70^{\circ} \mathrm{C}$ within four hours of collection and were thawed rapidly at $37^{\circ} \mathrm{C}$ within one hour before testing.

Table 2 Mean INR values of different instrument/reagent combinations. The mean INR values for the same instrument ${ }^{\star}$ and for all of the instruments $\dagger$ are ranked and compared in ascending order

\begin{tabular}{|c|c|c|c|c|c|}
\hline Instrument & Reagent & Mean & $S D$ & $\begin{array}{l}\text { Rank } \\
\text { within } \\
\text { group }\end{array}$ & $\begin{array}{l}\text { Overall } \\
\text { rank } \dagger\end{array}$ \\
\hline MLA Electra 750 & $\begin{array}{l}\text { Thromboplastin C Plus } \\
\text { Thromboplastin IS } \\
\text { Innovin } \\
\text { Simplastin Excel } \\
\text { Simplastin Excel S }\end{array}$ & $\begin{array}{l}2 \cdot 05 \\
2 \cdot 47 \\
2 \cdot 15 \\
2 \cdot 38 \\
2 \cdot 38\end{array}$ & $\begin{array}{l}0 \cdot 78 \\
0 \cdot 88 \\
0 \cdot 87 \\
0 \cdot 84 \\
0 \cdot 79\end{array}$ & $\begin{array}{l}2 \\
5 \\
1 \\
3 \\
4\end{array}$ & $\begin{array}{r}1 \\
12 \\
2 \\
7 \\
7\end{array}$ \\
\hline MLA Electra 900C & $\begin{array}{l}\text { Thromboplastin C Plus } \\
\text { Thromboplastin IS } \\
\text { Innovin } \\
\text { Simplastin Excel } \\
\text { Simplastin Excel S }\end{array}$ & $\begin{array}{l}2 \cdot 30 \\
2 \cdot 55 \\
2 \cdot 21 \\
2 \cdot 45 \\
2 \cdot 33\end{array}$ & $\begin{array}{l}0 \cdot 82 \\
0.92 \\
0 \cdot 88 \\
0 \cdot 85 \\
0 \cdot 76\end{array}$ & $\begin{array}{l}2 \\
5 \\
1 \\
4 \\
3\end{array}$ & $\begin{array}{c}4 \\
17 \\
3 \\
10 \cdot 5 \\
5\end{array}$ \\
\hline Coag-a-Mate X2 & $\begin{array}{l}\text { Thromboplastin C Plus } \\
\text { Thromboplastin IS } \\
\text { Innovin } \\
\text { Simplastin Excel } \\
\text { Simplastin Excel S }\end{array}$ & $\begin{array}{l}2 \cdot 53 \\
2 \cdot 65 \\
2 \cdot 38 \\
2 \cdot 54 \\
2 \cdot 41\end{array}$ & $\begin{array}{l}0.92 \\
0.97 \\
1.00 \\
0.93 \\
0.81\end{array}$ & $\begin{array}{l}2 \\
5 \\
1 \\
4 \\
3\end{array}$ & $\begin{array}{r}15 \\
19 \\
7 \\
16 \\
9\end{array}$ \\
\hline Coag-a-Mate XM & $\begin{array}{l}\text { Thromboplastin C Plus } \\
\text { Thromboplastin IS } \\
\text { Innovin } \\
\text { Simplastin Excel } \\
\text { Simplastin Excel S }\end{array}$ & $\begin{array}{l}2 \cdot 49 \\
2 \cdot 81 \\
2 \cdot 45 \\
2 \cdot 59 \\
2 \cdot 48\end{array}$ & $\begin{array}{l}0.98 \\
1.14 \\
1.08 \\
0.98 \\
0.86\end{array}$ & $\begin{array}{l}2 \\
5 \\
1 \\
4 \\
3\end{array}$ & $\begin{array}{l}14 \\
20 \\
10 \cdot 5 \\
18 \\
13\end{array}$ \\
\hline
\end{tabular}

Prothrombin time assays were performed by a single experienced technician to avoid inter-operator variation. The manufacturers' instructions for handling and usage of the thromboplastin reagents were followed and the ISI values recommended by the manufacturers for photo-optical instruments were used in the INR calculation. The same technician also checked the performance of each of the 20 test systems to ensure that the instruments met the manufacturer's specifications before testing. The precision of the systems was monitored with two commercial controls (Verify Normal and Verify Abnormal Level I, Organon Teknica Inc., Scarborough, Ontario, Canada) and by a pooled normal fresh plasma control prepared from four healthy volunteers. Control samples were assayed with every 15 to 20 test samples in all the test systems. All plasma samples of the patients and normal subjects were measured in duplicate. The mean values of the duplicate readings were used in the prothrombin time and INR calculations.

Conversion of prothrombin time results to INR values and the statistical analysis of the data were performed by a statistical computer program (SAS; SAS Institute Inc., Cary, North Carolina, USA).

\section{Results}

EFFECT OF THROMBOPLASTIN/

INSTRUMENT COMBINATIONS ON MEAN INR

VALUE

To assess the effect of thromboplastin/ instrument interaction on the accuracy of INR measurements, we compared the mean INR values for all combinations of thromboplastins and coagulometers used in the study (table 2). The use of Thromboplastin C Plus on MLA Electra 750 resulted in the lowest mean INR value (2.05), while the combination of Coag-a-Mate XM and Thromboplastin IS resulted in the highest mean INR value $(2 \cdot 81)$. The instruments from Organon Teknika Inc. (Coag-a-Mate X2 and Coag-aMate XM) showed consistently higher INR values than instruments from Baxter Diagnostic Inc. (MLA Electra 750 and MLA Electra 900C) (mean INR 2.50 and 2.56 v 2.28 and 2.37 , respectively). In contrast, reagents from Dade, Baxter Diagnostic Inc. yielded both the lowest (Innovin: $2 \cdot 30$ ) and the highest (Thromboplastin IS: $2 \cdot 62$ ) mean INR values, whereas the two reagents from Organon Teknika Inc. produced moderate mean INR values (Simplastin Excel: 2.49; Simplastin Excel S: 2.40). Thromboplastins with low ISI values did not confer less INR 
Figure 1 Comparison between the mean coefficient of variation (CV) of the groups defined by the mean INR ranges. $P T=$ prothrombin time.

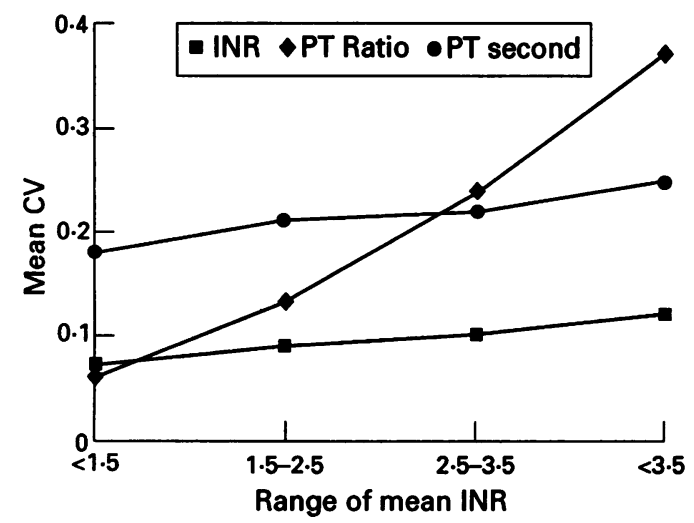

other instrument models to obtain the same INR value generated from the instrument chosen by the manufacturer. Thus, we assume that for each patient,

(PT ratio of a test system with manufacturer's chosen instrument) is is $=(\text { PT ratio of a test system with other instruments })^{\mathrm{E}-\mathrm{SI}}$

where PT is the prothrombin time and E-ISI is the estimated ISI value for the test system. Simple algebraic manipulation results in the following formula:

$$
\text { E-ISI }=\frac{\begin{array}{c}
\text { IS-ISI } \times \log (\text { PT ratio of a test system with } \\
\text { manufacturer's chosen instrument })
\end{array}}{\log (\text { PT ratio of a test system with other instruments })}
$$

The E-ISI values for all patients were then averaged to obtain an overall estimate of the ISI value (mean E-ISI) needed to produce the INR value corresponding to the instrument chosen by the manufacturer. Table 3 shows the mean E-ISI values of commercial thromboplastins on different coagulometers. As can be seen from table 3, the mean E-ISI values can be quite different from the IS-ISI values supplied by the manufacturers. In some cases the $95 \%$ confidence interval does not include the manufacturers' IS-ISI value-for example, Thromboplastin C Plus on MLA Electra 900C and Coag-a-Mate X2, Innovin on Coag-a-Mate XM, etc.

IMPACT OF IMPRECISION OF INR VALUES ON THERAPEUTIC DECISION

According to the recent consensus guidelines proposed by the American College of Chest Physicians, ${ }^{11}$ an INR of 2.0 to 3.0 is recommended for anticoagulant treatment of all forms of thromboembolic diseases (except for patients with mechanical prosthetic heart valves). We therefore used the discriminating values of INR of 2.0 and 3.0 to assess the impact of imprecision of INR values on the therapeutic decision-that is, adjustment of anticoagulant dose. To assess the degree of variability, the median INR value obtained from the 20 reagent/instrument combinations was plotted against the probability of the test systems reporting an INR value $>3.0$ in fig $2 A$ and $<2.0$ in fig $2 \mathrm{~B}$, respectively. As shown in fig $2 \mathrm{~A}$ between the range of median INR values of $2 \cdot 4$ to $3 \cdot 8$, a proportion of the test systems will generate an INR value $>3.0$ for a given patient. For example, for a patient with a median INR of 2.7 (point A), the probability of some test systems incorrectly stating that the INR value is $>3.0$ is $15 \%$. A patient with a

\begin{tabular}{|c|c|c|c|c|c|c|c|c|}
\hline \multirow[b]{2}{*}{ Reagent } & \multicolumn{2}{|c|}{$M L A$ Electra 750} & \multicolumn{2}{|c|}{$M L A$ Electra $900 C$} & \multicolumn{2}{|c|}{ Coag-a-Mate X2 } & \multicolumn{2}{|c|}{ Coag-a-Mate XM } \\
\hline & $\begin{array}{l}\text { Mean } \\
\text { estimated } \\
\text { ISI }\end{array}$ & $95 \% C I$ & $\begin{array}{l}\text { Mean } \\
\text { estimated } \\
\text { ISI }\end{array}$ & $95 \% C I$ & $\begin{array}{l}\text { Mean } \\
\text { estimated } \\
\text { ISI }\end{array}$ & $95 \% C I$ & $\begin{array}{l}\text { Mean } \\
\text { estimated } \\
\text { ISI }\end{array}$ & $95 \% C I$ \\
\hline Thromboplastin C Plus & $1 \cdot 96^{\star}$ & - & 1.47 & $(1 \cdot 33-1.62)$ & $1 \cdot 25$ & $(1 \cdot 08-1 \cdot 42)$ & $1 \cdot 74$ & $(1 \cdot 10-2 \cdot 37)$ \\
\hline Thromboplastin IS & $1 \cdot 33^{\star}$ & - & $1 \cdot 37$ & $(1 \cdot 27-1 \cdot 48)$ & $1 \cdot 38$ & $(1 \cdot 20-1 \cdot 56)$ & $1 \cdot 28$ & $(0.97-1.58)$ \\
\hline Innovin & 0.96 & $(0.92-0.99)$ & $0.92^{\star}$ & - & 0.83 & $(0 \cdot 81-0.85)$ & 0.81 & $(0.78-0.83)$ \\
\hline Simplastin Excel & $2 \cdot 05$ & $(1 \cdot 86-2 \cdot 24)$ & 2.07 & $(1 \cdot 98-2 \cdot 16)$ & $1 \cdot 97^{\star}$ & - & 1.93 & $(1 \cdot 88-1 \cdot 98)$ \\
\hline Simplastin Excel S & $1 \cdot 24$ & $(1 \cdot 17-1 \cdot 30)$ & $1 \cdot 32$ & $(1 \cdot 28-1 \cdot 35)$ & $1 \cdot 28^{\star}$ & - & $1 \cdot 19$ & $(1 \cdot 13-1 \cdot 25)$ \\
\hline
\end{tabular}
median INR of 3.5 (point B) will have $90 \%$ of

Table 3 The mean estimated ISI values of thrombobplastins and their $95 \%$ confidence intervals (CI)

${ }^{\star}$ Manufacturer specified IS-ISI value for photo-optical instruments. 

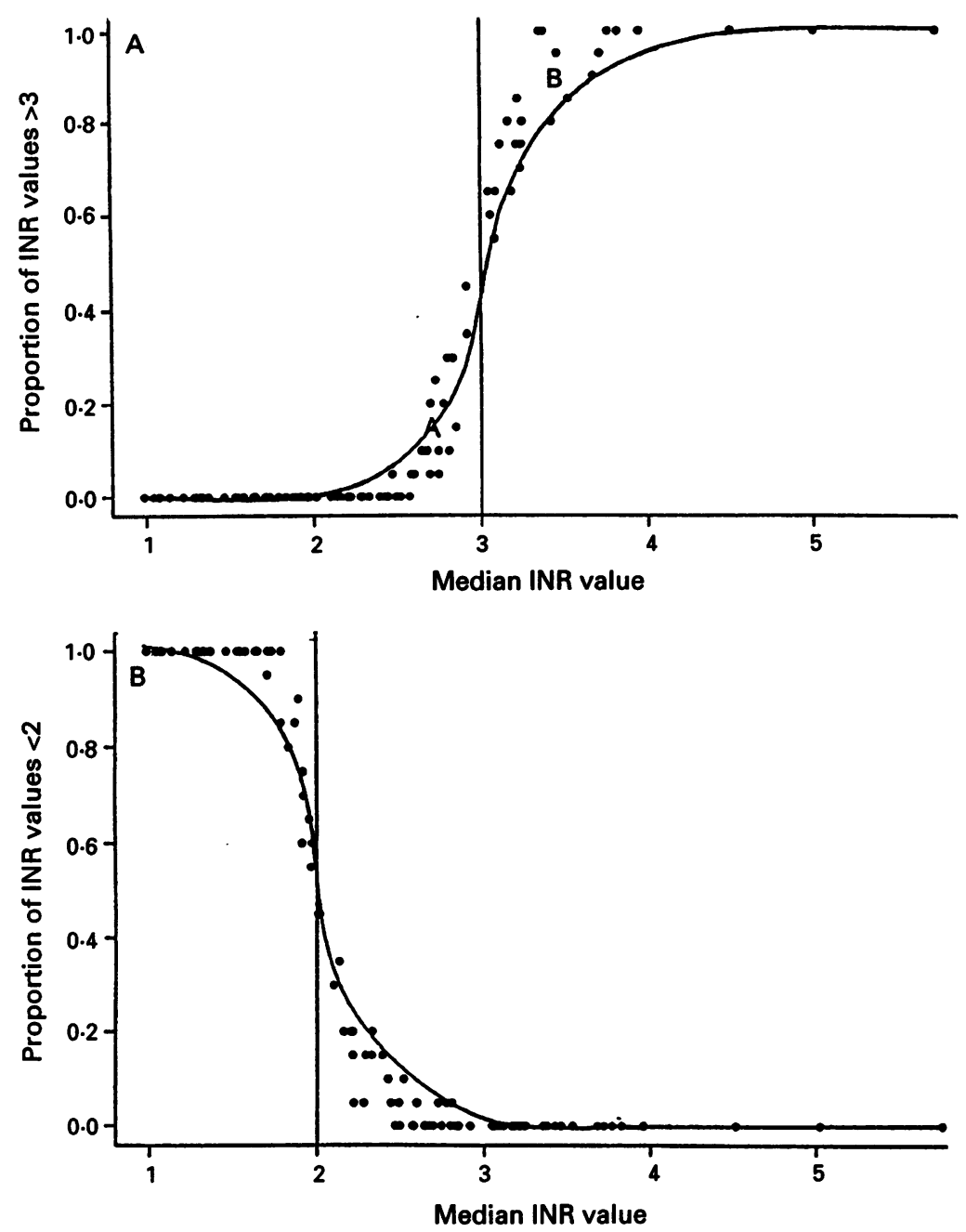

Figure 2 Proportion of the test systems reporting a median INR value $(A)>3.0$ and $(B)<2 \cdot 0$. See text for interpretation of points $A$ and $B$ in fig $2 A$. The dots in the graphs represent actual data points and the curves represent estimated probabilities over the range of median INRs. tem has been slow to gain recognition among clinicians and laboratory directors in North America. Undoubtedly, the INR system is more difficult for users to comprehend than the prothrombin time results given in seconds. A more forthright reason is the fact that interlaboratory imprecision of prothrombin time results had not been perceived as a major concern for clinicians in North America in the past. Until recently, the universal acceptance of insensitive commercial thromboplastin reagents with a limited range of responsiveness has minimised and "self regulated" the variability of the prothrombin time results generated by different laboratories. ${ }^{14}$ This is a reflection of insensitivity of the reagent rather than a result of increased accuracy. However, the problem of inconsistent reporting of prothrombin time results among laboratories has become greater in recent years with the promotion of the use of more sensitive commercial reagents. ${ }^{15}$

All of thromboplastins evaluated exhibited a wide range of instrument dependent fluctuation in sensitivity despite their IS-ISI values. Therefore, the provision of a single IS-ISI value for all photo-optical instruments is unlikely to resolve the problem of instrument dependent variation of the INR values. Nevertheless, despite its imperfection, our study has shown that the INR system is still superior to other forms of prothrombin time reporting formats to avoid extreme variations in prothrombin time results induced by the switch to more sensitive thromboplastins.

Our study was not designed to look at the overall precision of the prothrombin time assay. As all of our prothrombin time assays were performed in one laboratory and the INR values were obtained at only one time point for each patient, our results have not taken into consideration the differences between multiple measurements of each thromboplastin/instrument combination over time in different laboratories, the temporal fluctuations in INR values in patients, or differences between technicians. Furthermore, the scale of the study is also limited because only one model of coagulometer was used and therefore the variable effects of thromboplastin ISIs on individual instruments have not been accounted for. The overall interlaboratory imprecision of the INR system could conceivably be much higher than $10 \%$. Indeed, it is important to realise that the INR system is not the ultimate solution to the problem of imprecision and bias of automated prothrombin time assays. A long list of preanalytic and analytic technical factors (for example, sample preparation, storage, technical variation, instrument design, and performance, etc.) can adversely affect the performance of prothrombin time assays regardless of the postanalytic reporting format. Furthermore, some potential sources of imprecision are unique to the INR system. Besides thromboplastin/instrument interaction, variables such as the choice of the primary reference preparation for calibration ${ }^{16}$ or
Data from a recent College of American Pathologists survey has shown that about half of the laboratories in the United States are currently not reporting INR results. ${ }^{13}$

There are several reasons why the INR sys- 
the selection of mean "normal" plasma material $^{17}$ create notable variability.

Recent recommendations from several professional committees, including the American College of Chest Physicians, have suggested that the intensity of warfarin treatment should be lowered for many clinical indications. ${ }^{11}$ Our study has shown that there is a potential benefit in lowering the therapeutic range of anticoagulation - that is, the inter-laboratory variability of the prothrombin time assay can be slightly improved by the use of a lower therapeutic range. More studies, however, will be required to verify this postulate.

In summary, the development of the INR reporting system is a major advance towards standardisation of prothrombin time results for monitoring oral anticoagulant therapy. However, efforts should be made to improve the precision of the INR system when applied to the automated prothrombin time assays, especially when the reagents and instruments used in North American laboratories are so diverse. We feel that thromboplastin calibration should be maintained as a routine procedure whenever there is a switch in reagent or a change in instrumentation in each laboratory. Clarke et $a l^{18}$ showed that the most reliable way to correct the problem of imprecision caused by automation was to permit laboratories to determine their own corrected ISI on a range of calibrated plasmas. Many suppliers are now willing to provide user laboratories with a reference thromboplastin to conduct their own ISI calibration. Similarly, the College of American Pathologists is also working on the concept of providing reference calibrated plasmas for each laboratory that wants to check the accuracy of the ISI provided by the manufacturer. ${ }^{13}$ Failure to improve inter-laboratory precision will eventually undermine the usefulness of the INR system. It will also inevitably lead to erroneous adjustments of warfarin doses in an effort to maintain the narrow range of INR values regarded as optimal for anticoagulant control.

The authors would like to thank Dr A Mangal and Ms Sue Peterson and all the staff in the Hematology Department of Petro-McNair Clinical Laboratories for their encouragement and assistance. The authors would also like to thank Organon Teknika Inc. and Dada, Baxter Diagnostics Inc. for their support.

1 Taberner DA, Poller L, Thomson JM, Darby KV. Effect of international sensitivity index of thromboplastins on of international sensitivity index of thromboplastins on Precision of Interna

2 Poller L, Taberner DA, Thomson JM, Darby KV. Survey of prothrombin time in National External Quality Assessment Scheme exercises (1980-1987). f Clin Pathol 1988;41:361-4.

3 Thomson JM, Taberner DA, Poller L. Automation and prothrombin time: a United Kingdom field study of two widely used coagulometers. F Clin Pathol 1990;43: 679-84.

4 Poller L, Thomson JM, Taberner DA. Effect of automation on prothrombin time test in NSQAS surveys. f Clin Pathol 1989;42:97-100.

5 Barrow DA, Marnard JR. Standardization of the prothrombin time for monitoring oral anticoagulant therapy: The International Normalised Ratio. Dade therapy: The International Normalised Ratio. Dade Coagulation Technical Bulletin 19.

6 Houdijk WPM. INR: monitoring oral anticoagulant therapy with commercial thromboplastin: use of the International Normalised Ratio. Organon Teknica Technical Bulletin THR8806. Durham, North Carolina: Organon Teknika, 1988:6.

7 Ray MJ, Smith IR. The dependence of the International Sensitivity Index on the coagulometer used to perform the prothrombin time. Thromb Haemostas 1990;63: 424-6.

8 Becker DM, Humphries JE, Walker FB, DeMong LK, Bopp JS, Acker MN. Standardizing the prothrombin time: Calibrating coagulating instruments as well as thromboplastin. Arch Pathol Lab Med 1993;117:602-5.

$9 \mathrm{Ng} \mathrm{VL}$, Levin J, Corash C, Gottfried E. Failure of the International Normalized Ratio to generate consistent results within a local medical community. Am $\mathcal{F}$ Clin Pathol 1993;99:689-94.

10 National Committee for Clinical Laboratory Standards. Collection, transport and processing of blood specimens for coagulation testing and performance of coagulation for coagulation testing and performance of coagulation Villanova, Pennsylvania: 1991:11:1-5.

11 Hirsh J, Dalen JE, Deykin D, Poller L. Oral anticoagulants. Mechanism of action, clinical effectiveness and lants. Mechanism of action, clinical effectiveness and optimal therapeutic range. Third ACCP consensus con-
ference on antithrombotic therapy. Chest 1992;102 ference on antith

12 Ansell JE. Imprecision of prothrombin time monitoring of oral anticoagulation. A survey of hospital laboratories. Am $\mathcal{F}$ Clin Pathol 1992;98:237-9.

13 Check W. The INR gaining recognition. College of American Pathologists: CAP Today. 1993;7:1-26.

14 Poller L. Progress in standardization in anticoagulation control. Hematol Rev 1987;1:225-41.

15 Bussey HI, Force RW, Bianco TM, Leonard AD. Reliance on the prothrombin ratios causes significant errors in anticoagulant therapy. Arch Intern Med 1992;152: 278-82.

16 Poller L, Taberner DA, Thomson JM, Morris J, Mibashan RS, Shinton NK. Effect of the choice of WHO International Reference Preparation for thromboplastin on International Normalised Ratios. $\mathscr{f}$ Clin Pathol 1993; 46:64-6

17 Peters RHM, van den Besselaar AMHP, Olthuis FMFG. Determination of the mean normal prothrombin time for assessment of International Normalized Ratios. Thromb Haemostas 1991;64:442-5.

18 Clarke K, Taberner DA, Thomson JM, Morris JA, Poller L. Assessment of value of calibrated lyophilised plasmas to determine International Sensitivity Index for coagulometers. $¥$ Clin Pathol 1992;45:58-60. 